\title{
Correction to: Creating Business Applications with Office 365
}

\section{Techniques in SharePoint, PowerApps, Power BI, and More}

\section{Correction to:}

J. M. Rhodes, Creating Business Applications with Office 365: Techniques in SharePoint, PowerApps, Power BI, and More https://doi.org/10.1007/978-1-4842-5331-1

The book was inadvertently published with a smaller dimension for most of the chapter images and that has been replaced with the images in correct dimension.

The updated version of the book can be found at https://doi.org/10.1007/978-1-4842-5331-1 